\title{
The mitochondrial DNA copy number used as biomarker
}

\begin{abstract}
Mitochondria are organelles of eukaryotic cells which are responsible for the generation of metabolic energy. The mitochondrial matrix contains the mitochondrial genome, a molecule of circular DNA with 16,569 base pairs containing 37 genes that encode 13 proteins, 22 tRNAs, and 2 rRNAs. Each mitochondrion can have dozens of copies of its mitochondrial DNA depending on the cell type. However, variations in the mitochondrial DNA copy number have been associated with different pathological states including cancer and environmental exposure to some pollutants. Consequently, the mitochondrial DNA content represents a useful early biomarker to monitor premature changes in metabolic disorders due to external factors such as environmental pollutants or diseases. Therefore, in this minireview, the way to measure the mitochondrial DNA copy number by PCR as well as some relevant epidemiological studies are summarized.
\end{abstract}

Keywords: mitochondrial genome, copy number, biomarker
Volume 3 Issue 3 - 2018

\author{
Jorge Alejandro Alegría Torres \\ Laboratory of Pharmacy, Division of Natural and Exact Sciences, \\ University of Guanajuato, USA
}

Correspondence: Jorge Alejandro Alegría Torres, Laboratory of Pharmacy, Division of Natural and Exact Sciences, University of Guanajuato, Noria Alta s/n, C.P. 36050, Guanajuato, Gto, Mexico, USA, Email ja.alegriatorres@ugto.mx

Received: April 23, 2018 | Published: May 14, 2018

\section{Introduction}

The number of mitochondria per cell depends on the energy demand. For instance, muscle cells contain many mitochondria due to contractile activity. Likewise, sperm cells contain many mitochondria for motility. Thus, the estimated range goes from hundreds to one thousand mitochondria per cell, with 2 to 10 DNA copies per mitochondrion. ${ }^{1}$ Although there is a positive correlation between the number of mitochondria and the mitochondrial DNA copy number (mtDNAcn) ${ }^{2}$ it's noteworthy to mention that mtDNAcn is not always a reliable predictor for mitochondrial abundance. ${ }^{3}$ The mitochondrial genome has a particular vulnerability to oxidative stress because it lacks histones and nuclear DNA repair mechanisms. Besides, mitochondria are organelles that generate reactive oxygen species, so the damage to mitochondrial DNA will happen, including mutations and dysfunctional respiratory chains. ${ }^{4}$ Therefore, to compensate the loss by genotoxicity, an increase in the mtDNAcn occurs; however, new mitochondria will generate more oxidizing agents triggering a vicious cycle. ${ }^{2}$ In fact, several studies have found a positive correlation between biomarkers of oxidative DNA damage such as 8-hydroxy2'-deoxyguanosine (8-OHdG) and mtDNAcn, when both have been measured in different cells or tissues including leukocytes and cancer tissue biopsies. ${ }^{5-7}$

\section{How to estimate the mitochondrial DNA copy number}

The mtDNAcn can be measured by real-time PCR in different cell types, some of them are: white blood cells, embryo cells, sperm, and even in saliva samples. Further, mitochondrial DNA content can also be determined in cancer tissue biopsies where this biomarker is used to predict the prognosis of the disease. For example, a recent study found that increased mtDNAcn predicts poor prognosis of esophageal squamous cell carcinoma. ${ }^{8}$ Technically, a target fragment of the mitochondrial genome is amplified by real-time PCR and simultaneously quantified. The amplified product is compared with other PCR products from a single-copy nuclear gene; therefore, a relative quantification is obtained. ${ }^{9}$ Consequently, a standard curve is constructed with serial dilutions from a reference DNA composed of random samples. A DNA concentration range in nanograms for the curves is established considering the correlation coefficient, slope, and efficiency. The MT-ND1 gene is commonly used as target sequence in the mitochondrial genome while the globulin gene (HGB) as nuclear gene reference. The ratio MT-ND1/HGB is calculated for every sample expressed as cycle threshold value $(\mathrm{Ct})$ derived from the standard curve. ${ }^{10}$ Currently, new methodologies are being developed to determine the absolute mitochondrial DNA copy number using a digital PCR system which does not need to use references or standards, it is more sensitive than real-time PCR because detects small chances in the amplicons due to provides a linear response; and finally, the results are comparable between different studies because of it is an absolute quantification.

\section{Mitochondrial DNA copy number used as biomarker}

Although mitochondrial genome content is influenced by heredity, ${ }^{11}$ the environment and lifestyle also have effects on mtDNAcn. For example, exposure to several pollutants has been associated with changes in mtDNA content such as: benzene from vehicle and industrial emissions, ${ }^{12}$ inhalable particulate matter from air pollution, ${ }^{13,14}$ and polycyclic aromatic hydrocarbons from the incomplete combustion of organic matter. ${ }^{15,16}$ With respect to diet, the consumption of fructose and salt can modify the mtDNAcn. ${ }^{17}$ On the other hand, some cardiometabolic risk factors such as high blood pressure, dyslipidemia and obesity can also potentially modify the content of mtDNA. ${ }^{18,19}$ More studies have found an association between mtDNAcn and the risk of human cancers; for example, mtDNAcn variations have been associated with lymph node metastasis in patients with gastric cancer. ${ }^{20,21}$ In the same way, the variability in mtDNA content has been found in cervical cancer, ${ }^{22}$ while other reports indicate an increase in the susceptibility to develop esophageal adenocarcinoma, ${ }^{23}$ as well as a risk of breast and colorectal cancer. ${ }^{24,25}$ Further, elevated mtDNAcn could be a biomarker in pediatric acute lymphoblastic leukemia. ${ }^{26}$ Finally, recent research in the field of neurodegenerative diseases 
have shown that mtDNAcn is reduced in Parkinson's disease patients probably due to cumulative mitochondrial DNA mutations associated

Table I Changes in mitochondrial DNA copy number with oxidative stress. ${ }^{27,28}$ Factors related to mtDNAcn variations are summarized in (Table 1).

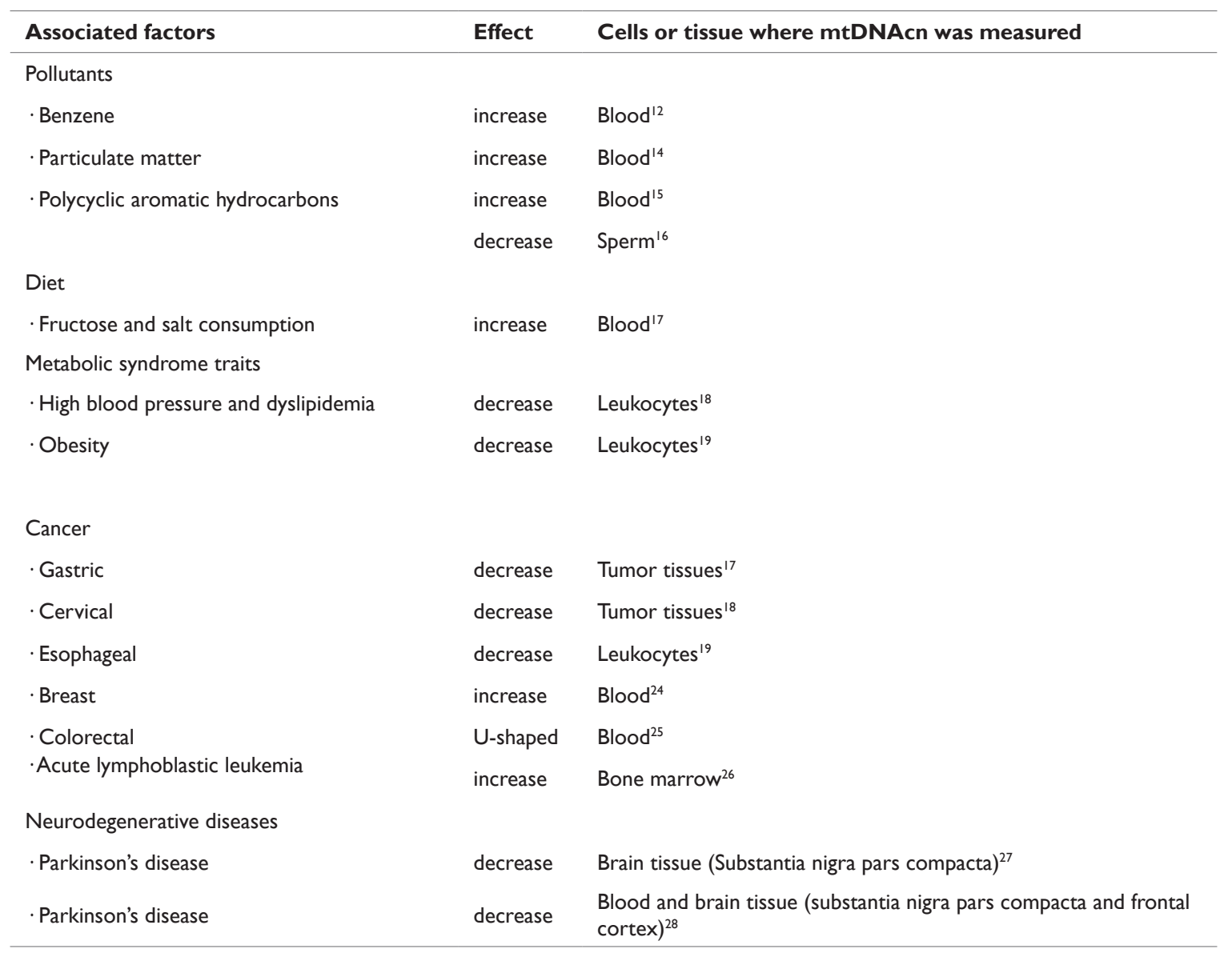

\section{Conclusion}

The mitochondrion is a primary source of reactive oxygen species and paradoxically a sensitive target to them, particularly the mitochondrial genome. Since mtDNAcn is an indicator of mitochondrial dysfunction, overproduction of reactive oxygen species and mitochondrial genome modifications, mtDNAcn changes can be a potential early biomarker of damage and dysfunction associated with intrinsic and extrinsic factors, it represents a potential predictive biomarker to monitor premature disorders at the molecular level related with metabolic imbalances, diseases, environmental insults as well as in the progression and prognosis of cancer.

\section{Acknowledgements}

None.

\section{Conflict of interest}

The author declares no conflict of interest.

\section{References}

1. Cavelier L, Johannisson A, Gyllensten U. Analysis of mtDNA copy number and composition of single mitochondrial particles using flow cytometry and PCR. Exp Cell Res. 2000;259(1):79-85.
2. Lee HC, Wei YH. Mitochondrial role in life and death of the cell. J Biomed Sci. 2000;7(1):2-15.

3. Cayci T, Kurt YG, Akgul EO, et al. Does mtDNA copy number mean mitochondrial abundance?. J Assist Reprod Genet. 2012;29(8):855.

4. Thompson LV. Oxidative stress, mitochondria and mtDNA-mutator mice. Exp Gerontol. 2006;4(12):1220-1222.

5. Qiu C, Hevner K, Abetew D, et al. Mitochondrial DNA copy number and oxidative DNA damage in placental tissues from gestational diabetes and control pregnancies: a pilot study. Clin Lab. 2013;59(5-6):655-660.

6. Liu CS, Tsai CS, Kuo CL, Chen HW, et al. Oxidative stress-related alteration of the copy number of mitochondrial DNA in human leukocytes. Free Radic Res. 2003;37(12):1307-1317.

7. Lin CS, Wang LS, Tsai CM, et al. Low copy number and low oxidative damage of mitochondrial DNA are associated with tumor progression in lung cancer tissues after neoadjuvant hemotherapy. Interact Cardiovasc Thorac Surg. (2008);7(6):954-958.

8. Li H, Tian Z, Zhang Y, et al. Increased copy number of mitochondrial DNA predicts poor prognosis of esophageal squamous cell carcinoma. Oncol Lett. 2018;15(1):1014-1020.

9. Hou P, Liu D, Shan Y, et al. Genetic alterations and their relationship in the phosphatidylinositol 3-kinase/Akt pathway in thyroid cancer. Clin Cancer Res. 2007;13(4):1161-1170. 
10. Alegría Torres JA, Velázquez Villafaña M, López Gutiérrez JM, et al. Association of leukocyte telomere length and mitochondrial DNA copy number in children from salamanca, Mexico. Genet Test Mol Biomarkers. 2016;20(11):654-659.

11. Xing J, Chen M, Wood CG, et al. Mitochondrial DNA content: its genetic heritability and association with renal cell carcinoma. J Natl Cancer Inst. 2008;100(15):1104-1112.

12. Carugno M, Pesatori AC, Dioni L, et al. Increased mitochondrial DNA copy number in occupations associated with low-dose benzene exposure. Environ Health Perspect. 2012;120(2):210-215.

13. Hou L, Zhang X, Dioni L, et al. Inhalable particulate matter and mitochondrial DNA copy number in highly exposed individuals in Beijing, China: a repeated-measure study. Particle and Fiber Toxicology. 2013;10:17.

14. Hou L, Zhu ZZ, Zhang X, et al. Airborne particulate matter and mitochondrial damage: a cross-sectional study. Environ Health. 2010;9:48.

15. Pavanello S, Dioni L, Hoxha M, et al. Mitochondrial DNA copy number and exposure to polycyclic aromatic hydrocarbons. Cancer Epidemiol Biomarkers Prev. 2013;22(10):1722-1729.

16. Ling X, Zhang G, Sun L, et al. Polycyclic aromatic hydrocarbons exposure decreased sperm mitochondrial DNA copy number: A cross-sectiona study (MARHCS) in Chongqing, China. Environ Pollut. 2017;220(Pt A):680-687.

17. Hernández Ríos R, Hernández Estrada S, Cruz Robles D, et al. Low fructose and low salt diets increase mitochondrial DNA in white blood cells of overweight subjects. Exp Clin Endocrinol Diabetes. 2013;121(9):535538.

18. Huang CH, Su SL, Hsieh MC, et al. Depleted leukocyte mitochondrial DNA copy number in metabolic syndrome. $J$ Atheroscler Thromb. 2011;18(10):867-873.
19. Hang D, Nan H, Kværner AS, et al. Longitudinal associations of lifetime adiposity with leukocyte telomere length and mitochondrial DNA copy number. Eur J Epidemiol. 2018.

20.Zhang G, Qu Y, Dang S, et al. Variable copy number of mitochondrial DNA (mtDNA) predicts worse prognosis in advanced gastric cancer patients. Diagn Pathol. 2013;8:173.

21. Wen SL, Zhang F, Feng S. Decreased copy number of mitochondrial DNA: A potential diagnostic criterion for gastric cancer. Oncol Lett. 2013;6(4):1098-1102.

22. Kabekkodu SP, Bhat S, Mascarenhas R, et al. Mitochondrial DNA variation analysis in cervical cancer. Mitochondrion. 2014;16:73-82.

23. Xu E, Sun W, Gu J, et al. Association of mitochondrial DNA copy number in peripheral blood leukocytes with risk of esophageal adenocarcinoma. Carcinogenesis. 2013;34(11):2521-2524.

24. Thyagarajan B, Wang R, Nelson H, et al. Mitochondrial DNA copy number is associated with breast cancer risk. PLoS One. 2013;8(6): e65968.

25. Thyagarajan B, Wang R, Barcelo H, et al. Mitochondrial copy number is associated with colorectal cancer risk. Cancer Epidemiol Biomarkers Prev. 2012;21(9):1574-1581.

26. Jain A, Bakhshi S, Thakkar H, et al. Elevated mitochondrial DNA copy numbers in pediatric acute lymphoblastic leukemia: A potential biomarker for predicting inferior survival. Pediatr Blood Cancer. 2013;65(3).

27. Coxhead J, Kurzawa Akanbi M, Hussain R, et al. Somatic mtDNA variation is an important component of Parkinson's disease. Neurobiol Aging. 2016;38(217):1-217.

28. Pyle A, Anugrha H, Kurzawa Akanbi M, et al. Reduced mitochondrial DNA copy number is a biomarker of Parkinson's disease. Neurobiol Aging. 2016;38(216):7-216. 